NOTA

333-342

\title{
ALCANCES METODOLÓGICOS PARA EL ESTUDIO DEL MUNDO DE LA VIDA DIARIA, DESDE LA PROPUESTA DE
} ALFRED SCHÜTZ

Methodological Approach for the Study of Daily Life, from Alfred Schütz's Proposal

Felipe Sáez Ardura * Arturo Vallejos-Romero **

Entre las múltiples propuestas teóricas que existen para explicar la realidad social, la de Alfred Schütz (2001) se presenta novedosa, clarificadora y precisa, ya que expone una descripción y explicación que integra las ciencias sociales y las ciencias de la cultura desde una fenomenología del mundo social. De este modo, las ciencias que aspiran a interpretar y explicar la acción y el pensamiento humanos "deben comenzar con una descripción de las estructuras fundamentales de lo precientífico, la realidad que parece evidente para los hombres que permanecen en la actitud natural" (p. 25).

Como programa filosófico de investigación, el enfoque de la nueva fenomenología social desarrollada por Schütz (1993) busca aclarar las diferencias sustanciales existentes entre las ciencias naturales, las del espíritu y las de la cultura, para desde ahí proponer un modelo explicativo para una nueva sociología de la comprensión. Esto tiene como finalidad otorgarle un estatus de "ciencia en sí" a las disciplinas encargadas de estudiar los fenómenos sociales, culturales, políticos y económicos (Weber, 1964). Para lograr esta tarea, el cientista social debe identificar empíricamente los presupuestos metodológicos que se tienen que considerar no solo al momento de hacer ciencia de la sociedad, sino que también cuando las personas se orientan de manera práctica en el mundo: de hecho, este último aspecto es el que le dicta los postulados esenciales a una fenomenología de lebenswelt. La tarea cotidiana del ser humano es intentar comprender lo que pasa a su alrededor, para ello debe tener desarrollada la capacidad del actuar racional y reflexivo respecto de este (Schütz, 1993; Leal, 2006).

En consecuencia, los alcances metodológicos que surgen desde la propuesta de la fenomenología del mundo social, desarrollada por el filósofo y sociólogo austríaco Alfred Schütz en el contexto epistémico referido a la distinción entre ciencias de la naturaleza y ciencias del espíritu, se conciben como propuestas de modelos explicativos para el mundo de la actitud natural que emergen directamente de un contexto epistémico referido a la distinción indicada. Los alcances de la propuesta permiten entender que una sociología comprensiva puede tener cabida en un contexto epistémico muy diferente al inicial, en donde la integración multidisciplinaria de las ciencias modernas caracteriza las actuales 
formas de generación de conocimiento ${ }^{1}$. Se ejemplifica lo anterior con la noción de riesgo, que si bien no se ha abordado desde la sociología comprensiva, podría tener cabida dentro del contexto de la propuesta schütziana.

En el contexto planteado, no deja de ser relevante comprender el alcance que tiene el postulado acerca de la estructura del mundo-vida propuesta por la sociología fenomenológica de Schütz para el desarrollo de las ciencias sociales, a pesar de que la distinción entre estas y las ciencias de la naturaleza, fundamentales para aquella tarea, en la actualidad ya haya aminorado dicha relevancia epistémica. La noción de "intersubjetividad" o "mundo intersubjetivo trascendental" propuesta por Husserl a las ciencias del mundo de la vida (Husserl, 1931; 1936), abre un horizonte de posibilidades a la teoría y a la práctica científico-social que estas no supieron enfrentar de forma rigurosa sino hasta bien entrada la primera mitad del siglo XX. La insistencia de ambos pensadores respecto de estas nociones a lo largo de todas sus obras se orientan, por parte de Husserl, a establecer una forma en donde la conciencia pura trascendental puede conocer el mundo en sus esencias, y por parte de Schütz (2001), a establecer las bases culturales con las que se edifican las ciencias sociales. Esta tarea tiene directa relación con el sentido común y con los criterios metodológicos que los mismos cientistas sociales deben desarrollar y mantener si es que quieren llevar adelante la tarea de comprender (científicamente) las estructuras del mundo de la vida diaria.

Fenomenológicamente, el estudio de las ciencias sociales, culturales y del hombre implica para Schütz hacerse cargo de una tradición que emana principalmente de los estudios de Husserl y su psicología trascendental, de la que toma como cimiento del pensamiento moderno un error en el que han incurrido principalmente las ciencias naturales, al perder la base de sentido del mundo en el que realiza sus descubrimientos e intervenciones centrales. Schütz (2003a), siguiendo a Husserl, indica que estas ciencias - principalmente la física matemática, la geometría y la lógica - han olvidado "la base de sentido a la cual (...) se refieren, o sea, el mundo de la vida" (p. 134). En otras palabras, las ciencias modernas han "olvidado" el mundo de la vida, lo que para Husserl implica olvidar las formas que adopta la realidad para los hombres y mujeres "despreocupados": "aquello que el ser humano ingenuo toma por la realidad natural no es el mundo objetivo de nuestras ciencias naturales modernas" (Schütz, 2003a, p. 133; Szilasi, 1959).

Muy por el contrario, el interés -y el éxito- de las ciencias naturales ha llevado a los cientistas sociales a enfocar metodológicamente sus estrategias y parámetros de éxito con los mismos procedimientos que las primeras, olvidando que un conjunto de reglas de procedimiento científico "tienen igual validez para todas las ciencias empíricas, ya se refiera a objetos de la naturaleza o a problemas humanos" (Schütz, 2003c, p. 72). Por ello, durante varios años los esfuerzos de los principales

\footnotetext{
${ }^{1} \mathrm{Si}$ bien se entiende que este punto es fundamental para enriquecer la discusión, no se considera como el objetivo de la presente nota.
} 
intelectuales se han orientado a tratar de emular el éxito de las disciplinas naturales, especialmente la física matemática y la lógica, por lo que Schütz afirma que las ciencias naturales, y las sociologías y antropologías positivistas subyacentes, buscarían una exactitud que los fenómenos culturales del mundo de la vida no permiten alcanzar (Schütz, 2003c.). Este es un alcance importante que realiza Schütz a los cientistas sociales: hacerse cargo de esta tradición, y en lo ideal, tratar de zafarse de ella, así como lo delineó el propio Husserl (1913, p. 46-63).

Una vez realizada esta tarea, correspondería al cientista social reconocer el origen esencial de este conocimiento científico acerca del mundo, sea aquel natural o cultural: la subjetividad trascendental (Husserl, 128, p. 126), "única que posee el sentido óntico del ser absoluto" (Schütz, 2003a, p. 129). Agregará el pensador de Viena de manera insistente que es en esta subjetividad trascendental donde surgirán las coordenadas temporoespaciales, tanto del investigador como de la persona en actitud natural, y que el pensamiento solo puede ser instituido por ella para lograr reconocer las estructuras del mundo de la vida cotidiana (Schütz, 2001).

Producido ello, en lo relativo a la subjetividad trascendental, conviene considerar este aspecto mundano como una práctica, una realización, sobre todo si se piensa que las personas que generan los profundos fenómenos sociales que estudian las ciencias culturales viven su vida, "de modo concreto y uniforme", cuyo ámbito finito de sentido (sedimentado) es referido como "un ámbito de actividad práctica, que posee desde el primer momento significaciones humanas" (Schütz, 2003a, p. 133)².

Complementa este análisis husserliano el poder pensar en esta subjetividad trascendental como una intersubjetividad de sentido común (Schütz, 1993; Leal, 2001), no problemático (Schütz, 2001, p. 29), que distribuido socialmente se emplea para poder actuar en el mundo, para "estar ahí"3. Esta tarea, vivir en un mundo

\footnotetext{
${ }^{2}$ Precisamente de estos principios metodológicos arranca Harold Garfinkel (1967) para fundar la escuela etnometodológica. Este insistía en que estudiar los etnométodos apunta "a la investigación de las propiedades racionales de las expresiones indexicales y otras formas contingentes para la organización de las prácticas de la vida diaria" (Garfinkel, 1967, p. 1; Coulón, 1988, p. 32). Nociones como práctica o realización (métodos), indexicalidad (persona, espacio y tiempo en un contexto), reflexividad (capacidad para comprender a los otros, mi propia acción y expresar dicha comprensión), descripción o account (posibilidad y capacidad de referirse a lo que uno mismo hace) y lenguaje vernáculo (idiosincrasia y pertenencia a una etnia de sentido), forman parte de este paradigma de la sociología no tradicional, potente al momento de querer estudiar los fenómenos sociales. Schütz en reiterados pasajes de sus obras insistió en esta innegable capacidad práctica de las personas, incluso de las organizaciones y constructos institucionales que emergen de las relaciones recíprocamente orientadas: "la sociedad y la comunidad, el Estado y las colectividades, son experimentados por mí en el anonimato de "alguien", de "personas", que hacen precisamente lo que "se hace" (Schütz, 2003d, p. 146). En otro trabajo agrega, respecto de esta eminente realidad práctica, que "no existe ningún motivo para que la persona ingenua suscite la cuestión trascendental concerniente a la realidad del mundo o a la realidad del alter ego" (Schütz, 2003a, p. 139).

3 "Mi subjetividad trascendental, en cuyas actividades se constituye este mundo, debe estar relacionada desde el comienzo con otras subjetividades, con relación a cuyas actividades autoriza y rectifica su propia actividad. Y a este mundo de la vida, que es caracterizado como el mundo de la vida único y unitario de todos nosotros,
} 
intersubjetivo, tiene que desarrollarla no solo la persona "ingenua" o "desinteresada", sino también el propio científico social que desee conocer las estructuras del mundo de la vida diaria. Este mundo intersubjetivo permite entender que los objetos de pensamiento que el cientista social puede elaborar para comprender esta realidad se deben basar en los objetos construidos por el "pensamiento de sentido común de hombres que viven su existencia cotidiana dentro de su mundo social" (Schütz, 2003c, p. 80; Leal, 2003). Vale decir, debe existir una mutua interconexión y retroalimentación entre ambos tipos relevantes de sentido, en lo que Giddens (1987) denominará posteriormente "doble hermenéutica". Como se puede apreciar, ambos ejes de coordenadas surgen precisamente de la esfera precientífica de la realidad idiosincrática que cada actor vive en su día a día ${ }^{4}$.

Para Schütz (2003c), el científico social debe modificar su eje de coordenadas, pero también debe mantener vigente un principio metodológico claro, a saber, que "son las construcciones del primer nivel, sobre las cuales deben ser erigidas las construcciones del segundo nivel de las ciencias sociales" (p. 82). Vale decir, la labor del científico social se basa en un intercambio regular e insistente "entre la práctica de la vida cotidiana y la conceptualización de las ciencias sociales" (p. 84). Esta reconstrucción del sentido intersubjetivo de las personas y los grupos sociales, que para el cientista social se reconoce como un campo observacional con "un significado específico y una estructura de significatividades para los seres humanos que viven, actúan y piensan dentro de él” (p. 79), implica destacar las formas interpretativas por las que la gente vive en un "ahí" y un "ahora" constante en su mundo de la vida cotidiana.

A diferencia de Husserl $(1913,1928)$, quien se adentra en los aspectos filosóficos de la intersubjetividad mediante la definición de la subjetividad trascendental, las ciencias sociales y humanas, a juicio de Schütz, se deben adentrar en la estructura del lebenswelt tal como la experimentan las personas en su actitud natural, para ello se deben emplear diversas formas de observación social (Schütz, 1993), nociones que toma de Max Weber para el análisis de las relaciones sociales. De este modo, toda ciencia social que se reconozca como disciplina empírica debe reconocer los postulados de la fenomenología trascendental husserliana, pero metodológicamente desarrollar una fenomenología de la actitud natural (Schütz, 2003d, p. 150). Esto quiere decir, según Schütz, que hay que reconocer los principios regulares por los que la gente en su vida diaria mantiene sus relaciones sociales (Schütz, 1993; Leal, 2001). Estas relaciones

pertenecen en verdad todos los fenómenos de la vida social, desde la simple relación 'Tú' hasta los más diversos tipos de comunicaciones sociales" (Schütz, La fenomenología, 128).

4 "La sociología, no obstante, se ocupa de un universo que ya está constituido dentro de los marcos de significado por los actores sociales mismos, y los reinterpreta dentro de sus propios esquemas teóricos, mediando el lenguaje corriente y técnico. Esta doble hermenéutica es de una considerable complejidad, puesto que su conexión no es meramente unívoca (como Schütz parece sugerir); hay un continuo deslizamiento de los conceptos construidos en sociología, mediante el cual se apropian de ellos los individuos para el análisis de cuya conducta fueron originalmente acuñados, y por consiguiente tienden a convertirse en rasgos integrales de esa conducta" (Giddens, 166). 
alcanzan diversos grados de proximidad y anonimia, y es desde el eje de coordenadas centrales que tiene la sociedad, vale decir, la persona, desde la que emergen todas las demás formas de relaciones sociales, estas derivan a su vez de la interacción cara a cara o "relación nosotros" (Schütz, 1993, con base en Weber). Es interesante resaltar que "solo en ese ámbito podemos ser comprendidos por nuestros semejantes, y solo en él podemos actuar junto con ellos" (Schütz, 2001, p. 25).

Schütz logra otorgarle un interés central a la base cultural del mundo de la vida, a una base de sentido que es culturalmente entendida, interpretada y comprendida por los seres humanos. Atribuir un carácter cultural a esta esfera mundana refleja un interés que aquel que esté empeñado en estudiar sus estructuras debe reconocer como un "universo de significación para, es decir, una estructura de sentido (Sinnzusammenhang)" (Schütz, 2003a, p. 137). Por cierto que este mundo de significaciones, que está sedimentado, que está impregnado de tipificaciones, que construye el sentido intersubjetivo, se origina en el sí mismo (Mead, 1973) y está orientado "con respecto a un 'punto cero' u origen de coordenadas (Nullglied), o sea, una personalidad" (Schütz, 2003a, p. 132).

Debido a lo anterior, una afirmación importante en la que insiste regularmente Schütz en relación con el estudio científico del mundo intersubjetivo se orienta a resaltar, reconocer y perfeccionar el método-técnica de la reducción fenomenológica, esencial al momento de realizar una ciencia objetiva de la subjetividad e intersubjetividad del mundo de la vida (Schütz, 2003a; 2003b; 2003d).

La reducción fenomenológica ${ }^{5}$ es una actitud que asume el investigador respecto del mundo empírico, al que Schütz denomina el "observador desinteresado". Es precisamente esta actitud de desinterés respecto del mundo cultural lo que la diferencia de aquellos que considera sujetos de análisis, es decir, la persona que vive "ingenuamente" su mundo de la vida diaria (Schütz, 2003a, p. 140), y que como se mencionó anteriormente, posee un interés eminentemente práctico en él. Garfinkel (1967) dirá que es un interés basado en realizaciones prácticas. Esta técnica permite tomar cierta distancia de los objetos que observamos y llegar a elaboraciones explicativas acerca del funcionamiento de las estructuras del mundo social, cuya validez se asienta en la esfera mundana no problemática. Esta idea implicaría un trabajo de manera intencionada y sistemática de "poner el mundo entre paréntesis" y activar así el proceso de la duda filosófica, que tiene utilidad para alcanzar un nivel de certeza incuestionable que se sitúe más allá del dominio de la mera creencia, es decir, que se oriente a revelar el ámbito puro de la conciencia (Schütz, 2003a).

\footnotetext{
${ }^{5}$ Schütz (2003b) concibe la reducción fenomenológica con los siguientes términos: "el fenomenólogo no niega la existencia del mundo externo, pero, para sus fines analíticos, decide suspender la creencia en su existencia; es decir, abstenerse intencional y sistemáticamente de todo juicio relacionado directa o indirectamente con la existencia del mundo externo" (p. 115).
} 
Es a esto a lo que apunta la idea de epoché $e^{6}$ la que sería un resultado de la aplicación de la técnica de la reducción, o mejor dicho, otra cara de la misma medalla, ya que implica suspender nuestras creencias y nociones acerca de la existencia del mundo exterior en sus diversos aspectos, incluyendo no solo nuestras afirmaciones acerca de este mundo, sino también las proposiciones de todas las ciencias que se refieren a la existencia de la realidad como requisito fundamental en nuestro rol como observadores (Schütz, 2003a).

Si bien Schütz se sumerge en una primera etapa en el estudio de la conciencia como elemento principal para abordar el problema de la realidad social desde el mundo de la vida cotidiana, los aportes desde los conceptos antes mencionados permiten el traslado a un estadio que supera lo subjetivo, y como señala Leal (2007), hace un llamado al investigador, que también es un ego, a que suspenda su juicio personal (valores, prenociones, interpretaciones diversas, etc.) acerca de la realidad que lo rodea.

Estos aspectos, entre otros, serían los que dotan de importancia a las reflexiones del autor en torno a su método-teoría para las ciencias sociales. Respecto de la epoché, Lyotard (1989) señala que poner entre paréntesis consiste "en primer lugar, en dejar atrás una cultura, una historia, en retomar todo saber remontándose a un no saber radical" ( $\mathrm{p}$. 10). Por esta razón, metodológicamente el investigador debe suspender los denominados juicios críticos respecto del mundo en su esfera de actitud natural, activar la duda filosófica cartesiana, y junto con ello lograr identificar en el ámbito de la esfera reducida -Husserl diría: en el ámbito puro de la conciencia- los componentes esenciales, fundamentales, del mundo de la vida. Como dice el propio Husserl, "dentro de la actitud teórica que llamamos "natural", queda, pues, designado el horizonte entero de las indagaciones posibles con una sola palabra: es el mundo" (Husserl, 1913, p. 17).

Esto es, se deja en suspenso todo lo que se refiere a las tomas de posición por parte del yo que reflexiona filosóficamente, lo que no quiere decir que las mismas desaparezcan de su campo de experiencia del sujeto consciente, sino que más bien se "desconectan" desde lo que se refiere a la actitud básica para ver el mundo, con el objetivo de llegar a lo que es la descripción de las puras vivencias de una subjetividad pura, lo que no se puede alcanzar en la actitud natural.

Esto alcances metodológicos propuestos por Schütz pueden tener una aplicación interesante en un área específica de las actuales ciencias sociales, las que, por decirlo de algún modo, han dejado de lado los aportes de este importante paradigma: el enfoque de los riesgos sociales, y en específico, lo que se refiere a la sociología del riesgo, como ha sido elaborada principalmente por Beck (1998a,

\footnotetext{
${ }^{6} \mathrm{Si}$ bien no hay un acuerdo generalizado respecto de si la reducción y la epojé son o no sinónimos, para Schütz la cuestión está clara: "para develar esta esfera de la subjetividad trascendental, el filósofo, al comenzar su meditación dentro de la actitud natural, debe emprender ese cambio de actitud que Husserl llama epojé fenomenológica o reducción fenomenológica trascendental" (Schütz, 2003a, p. 129).
} 
1998b, 2002, 2008), o la teoría de la sociedad del riesgo mundial ${ }^{7}$.

Urteaga con Eizaguirre (2013), por su parte, son bastante enfáticos al afirmar no solo el carácter "eurocéntrico" del riesgo, sino que también al indicar sin temor a equívocos que en estos enfoques desaparece cualquier intento "por entender la capacidad de agencia de los individuos en su comprensión y articulación de los discursos y de las prácticas ligadas al riesgo" (p. 158).

Para estos, una sociología del riesgo debiera también interesarse "por el significado de las actividades realizadas por los individuos en su vida personal, profesional o de ocio que mantienen una relación con el riesgo" (Urteaga y Eizaguirre, 2013, p. 163), enfoque que se debiera complementar con las posturas del riesgo en las sociedades contemporáneas que defienden una visión de carácter "objetivista", como lo haría Beck, y "culturalista" (Douglas, 1996).

Apostando por un enfoque que llevaría a integrar al estudio de los riesgos una sociología comprensiva, afirman que las investigaciones del riesgo en las sociedades periféricas, aunque también en las centrales, debieran resaltar la posibilidad de captar los elementos esenciales de las experiencias y significados que revisten los riesgos actuales para la gente, "en tanto que son variables explicativas sobre el modo en que operan estos significados como parte de la subjetividad y de las relaciones sociales de los individuos" (Urteaga y Eizaguirre, 2013, p. 163).

No cabe duda que la fenomenología de Schütz, como se propone para el estudio de los aspectos referidos al sentido intersubjetivo en el mundo de la vida diaria, puede ser una herramienta interesantísima para enfrentar este emergente paradigma de los riesgos en la sociedad moderna, el que si bien es cierto ha "colonizado" a nivel epistémico amplios campos del saber científico-social, ha demostrado una tendencia a relegar a un segundo plano los significados de los seres humanos respecto de las complejas incertidumbres que se generan en el seno de las propias sociedades complejas.

Los alcances de la fenomenología social que se han indicado en los párrafos anteriores se orientan a entregar un programa metodológico para las ciencias de la cultura, para ello no solo requiere desvincularse de la larga tradición positivista de la ciencia tradicional, sino que además reforzar la importancia de la fenomenología trascendental propuesta por Husserl para ampliar el campo de aplicación de este relevante enfoque, que sin dudas ha cambiado el rumbo de las ciencias humanas y sociales.

\footnotetext{
${ }^{7}$ En el complejo orden social contemporáneo, se diluyen las responsabilidades y cada respuesta ante riesgos y peligros que se presentan se vuelve más compleja aún (1998a). Por esto, al problematizarlo y tratar de abordarlo el observador se encuentra con un alto y creciente nivel de "inseguridad" en la sociedad, la que propicia nuevas formas para responder a ellos. Producto de la inseguridad social ante los riesgos autoproducidos, las sociedades se tensionan y atrofian en sus formas reflexivas de decisión y de acción, bloqueando la observación de las amenazas e imposibilitando de manera crítica su anticipación. De este modo se podría afirmar que lo que falla es la capacidad de la racionalidad moderna para estructurar respuestas adecuadas ante los complejos riesgos y peligros que generan las sociedades contemporáneas.
} 
Para alcanzar este objetivo, por medio del "giro sociológico" de la filosofía trascendental de Husserl, Schütz se aventura a desarrollar a lo largo de todo su trabajo ciertas nociones que, si bien no elaboró sistemáticamente, las dejó perfiladas mediante su concepción respecto del mundo de la actitud natural o su "tesis de la actitud natural"' (Schütz, 1993, 2001).

Si se analizan estas prescripciones, todas las sugerencias seleccionadas y los argumentos entregados por el filósofo a lo largo de su carrera tienen la finalidad de reconocer y distanciarse de las ciencias sociales basadas en las ciencias naturales. En todas estas sugerencias se identifica un hilo conductor esencial que tiene como objetivo transformar este profundo esquema analítico entregado por Husserl para la filosofia, en un esquema analítico para las ciencias sociales o ciencias culturales, como prefiere denominarles Schütz.

En este camino recorrido se encuentra con algunos obstáculos, gran parte de ellos de naturaleza metodológica, y que se sintetizan en la interrogante respecto de "¿cómo lograr una construcción científica del mundo social estudiando la subjetividad e intersubjetividad en esta misma esfera mundana?". No es fácil dar respuesta a esta pregunta, no obstante, y con bastante éxito, lo que hace Schütz es siempre resaltar la importancia del sentido humano (intersubjetivo) tal y como se forma y se sedimenta en la esfera de la actitud natural, vale decir, el reconocer cómo funcionan las estructuras del mundo social desde el punto de vista de las personas que viven "ingenuamente" en este.

Por tanto, para levantar un conocimiento certero acerca de estas estructuras es que se hace necesario determinar las formas en las que los cientistas sociales pueden abordar el objeto propio de sus investigaciones, es decir, las relaciones sociales en el mundo de la vida: una metodología adecuada para aquello. Los años que Schütz dedicó a esbozar estos principios marcaron sin duda alguna un antes y un después en las ciencias sociales contemporáneas. Es responsabilidad de los propios cientistas sociales ampliar los campos de aplicación de esta fenomenología del mundo social, tal cual se presenta este mundo en las actuales sociedades modernas.

Universidad Autónoma de Chile* Av. Alemania 01090, Temuco (Chile) f.saez05@ufromail.cl

$\operatorname{Dr}(\mathrm{C}$ en Ciencias Sociales, UFRO Av. Francisco Salazar 01145, Temuco (Chile)

Universidad de La Frontera** Núcleo de Ciencias Sociales y Humanidades

Doctorado en Ciencias Sociales Av. Francisco Salazar 01145, Temuco (Chile) arturo.vallejos@ufrontera.cl 


\section{OBRAS CITADAS}

Beck, Ulrich. (2008). La sociedad del riesgo mundial. En busca de la seguridad perdida. Barcelona: Paidós.

— (2002). La sociedad del riesgo global. Madrid: Siglo XXI.

(1998a). La sociedad del riesgo. Hacia una nueva modernidad. Barcelona: Paidós.

— (1998b). ¿Qué es la Globalización? Falacias del Globalismo. Respuestas a la Globalización. Barcelona: Paidós.

_ (1997). Teoría de la sociedad del riesgo reformulada. Revista Chilena de Temas Sociológicos, № 4-5, 11-42.

Coulón, Alain. (1988). La etnometodología. España: Cátedra.

Douglas, Mary. (1996). La aceptabilidad del riesgo según las ciencias sociales. Barcelona: Paidós.

Garfinkel, Harold. (1967). Studies in Ethnometodology. New Jersey: Prentrice Hall.

Giddens, Anthony. (1987). Las nuevas reglas del método sociológico. Crítica positiva de las sociologías interpretativas. Buenos Aires: Amorrortu.

Husserl, Edmund. (1936). La crisis de las ciencias europeas y la filosofia trascendental. Evanston: Northwestern University.

— (1931). Meditaciones cartesianas. Una introducción a la fenomenología. París: Armand Colin.

_ (1928). Fenomenología de la conciencia del tiempo inmanente. Buenos Aires: Nova.

— (1913). Ideas relativas a una fenomenología pura y una filosofia fenomenológica. México: Fondo de Cultura Económica.

Leal, Rubén. (2007). "Aportes teórico-metodológicos de la fenomenología al desarrollo cualitativo de las ciencias sociales en Alfred Schütz". ALPHA, $\mathrm{N}^{\circ}$ $25,215-225$.

— (2006). "La sociología interpretativa de Alfred Schütz. Reflexiones en torno a un planteamiento epistemológico cualitativo". ALPHA, $\mathrm{N}^{\circ} 23,201-213$.

— (2003). "Análisis del desarrollo social intersubjetivo desde las nociones de mundo de la vida y mundo de la ciencia, propuestos por Alfred Schütz". ALPHA, $\mathrm{N}^{\circ} 19,263-275$.

— (2001). "El problema de la intersubjetividad. Aproximaciones a la propuesta epistemológica de Alfred Schütz". ALPHA, 우 17, 215-229.

Lyotard, Jean François. (1989). La fenomenología. Barcelona: Paidós.

Mead, George Herbert. (1973). Espiritu, persona y sociedad. Desde el punto de vista del conductismo social. Barcelona: Paidós.

Schütz, Alfred. (2003a). "La fenomenología y las ciencias sociales". En: El problema de la realidad social. Escritos I. Buenos Aires: Amorrortu, 126-142. 
— (2003b). "Conceptos fundamentales de la fenomenología". En: El problema de la realidad social. Escritos I. Buenos Aires: Amorrortu, 111-125.

— (2003c). "Formación de conceptos en ciencias sociales". En: El problema de la realidad social. Escritos I. Buenos Aires: Amorrortu, 71-85.

_ (2003d). "Importancia de Husserl para las ciencias sociales". En: El problema de la realidad social. Escritos I. Buenos Aires: Amorrortu, 143-150.

— (2001). "El mundo de la vida cotidiana y la actitud natural". En: Las estructuras del mundo de la vida. Buenos Aires: Amorrortu, 25-40.

— (1993). La construcción significativa del mundo social. Introducción a la sociología comprensiva. Barcelona: Paidós.

Szilasi, Wilhelm. (1959). Introducción a la fenomenología de Husserl. Buenos Aires: Amorrortu.

Urteaga, Eguzki y Eizaguirre, Andoni. (2013). "La construcción social del riesgo". Empiria. Revista de Metodología de las Ciencias Sociales, $\mathrm{N}^{\circ} 25,147-170$.

Weber, Max. (1964). Economía y sociedad. Esbozo de sociología comprensiva. México: Fondo de Cultura Económica. 